\title{
On a computational approach to micro- and macro-modelling of damage in brittle and quasi-brittle materials
}

\author{
Jiř́ Vala \\ Brno University of Technology, Faculty of Civil Engineering, \\ Institute of Mathematics and Descriptive Geometry \\ 60200 Brno, Veveří 331/95, Czech Republic \\ Vladislav Kozák \\ Czech Academy of Sciences, Institute of Physics of Materials \\ 61662 Brno, Žižkova 513/22, Czech Republic \\ Petra Jarošová \\ Secondary Vocational School \\ 69301 Hustopeče, Masarykovo nám. 136/1, Czech Republic
}

Received: September 10, 2020. Revised: October 15, 2020. Accepted: October 19, 2020.

Published: October 21, 2020.

\begin{abstract}
Computational modelling of damage in brittle and quasi-brittle materials needs some coupling between micro- and macroscopic crack initiation and evolution, up to their nonnegligible softening behaviour. Most such approaches contain ad hoc evaluations, with some physical and engineering motivations, namely those connected with massive application of steel fibre-reinforced concrete and similar composites in building projects, but without any proper mathematical existence and convergence analysis for the time development of damage. This paper presents a possibility of such deterministic analysis on a selected model problem of structural dynamics, supplied by comments to useful directions of generalization. Several application examples document the feasibility of such approach, up to its software implementation and real data validation.
\end{abstract}

Keywords- Nonlocal viscoelasticity, brittle and quasi-brittle materials, micro- and macrofracture, extended finite element method, method of discretization in time, Rothe sequences.

\section{INTRODUCTION}

Computational prediction of fracture and damage of materials and structures under mechanical, thermal and other loads belongs to serious problems in many engineering applications. Most considerations on physical mechanisms of creation and propagation of microfractured zones, macro-cracking, etc., give only quali- tative results for idealized materials and processes, observable during well-considered laboratory experiments, not reliable quantitative results for real materials and composites, as bearing parts of buildings or engineering structures. Usual manifestation of fracture are: i) discrete crack discontinuities in brittle materials like glass or welds in metal structures, ii) shear (localization) bands in elasto-plastic ductile metals and similar materials, or iii) fracture process zones with numerous particular microand macro-cracks, typical for quasi-brittle materials, in comparison with i); for the more detailed classification see [1], for the attempt to develop a unified physical and mathematical theory cf. 2]. Especially in iii) two stages of damage can be recognized: iii-a) formation of micro-fractured zones, reducing the stiffness of a structure, iii-b) creation of macro-cracks, whose later opening and closing is conditioned by the cohesive characteristics of new interfaces.

In general, from the point of view of computational practice, in all cases i), ii), iii) the presence of some irregular boundaries and interfaces at several scales should be respected. Namely in the probably most frequent building application of fibre-reinforced cementitious composites, containing fibres prepared from steel or some advanced materials, corresponding to iii), at least the following scales should be distinguished: 1) matrix particles (at $10^{-3} \mathrm{~m}$ ), 2) hardening fibres (at $10^{-2} \mathrm{~m}$ ) and 3) laboratory samples (at $10^{-1} \mathrm{~m}$ ) or real structures in situ (even greater). Thus any quantitative computational modelling or simulation of i), ii) or iii) is conditioned by the reasonable identification of geometrical parameters like volume fraction and fibre orientation by 1), 2), 3), together with a suitable aet of mechanical characteristics of particular components and interfaces. 
In most cases some non-destructive or low-invasive testing approaches are required, especially in the case of fibre-reinforced composites a) X-ray (roentgenographic) planar images, supplied by image processing, supported by the FFT (Fast Fourier Transform) algorithm, b) 3dimensional images from computer tomography, c) indirect measurements of changes in a (nearly) stationary magnetic field (applying e.g. the Hall probe), d) indirect measurements of changes in a harmonic electromagnetic field, etc., as demonstrated in 3], containing numerous further references.

The extensive use of brittle matrix composite materials requires also appropriate computational models to describe, with adequate accuracy, their mechanical behaviour. From a micromechanical model some macroscopic constitutive equations are derived for intentionally or random oriented fibres by [4], [5], [6] and 7], accounting for such physical processes as matrix / fibre debonding and fibre rupture. One of possible ways is to adopt a discontinuous-like finite element approach to the lattice model by 8. An alternative approach refers to special constitutive relations, inspired by continuum mechanics, where crack opening and contact surface sliding are included into the model of plastic damage, using smeared cracking by 9, 10 or 11, together with mesh objective strain localization due to material softening, referring to the thermodynamically irreversible continuum damage mechanics by [12], in particular that leading to a smeared representation of the crack path by 13 . At least for the practically significant application of self-compacting concrete, supported by both experimental methods and numerical simulations, smeared cracking can be combined with Monte Carlo simulations, which results in the variational multiscale cohesive method by [14, whose various implementations differs in the choice of basis functions.

Some authors refer to the non-negligible nondeterministic both input data and relevant physical processes, which leads them to the attempts to handle the evolution of damage by switching to stochastic considerations, genetic algorithms or other soft computing approaches like [15] or 16], to statistical physics by 17], or to computational peridynamics, avoiding all gradient evaluations, as discussed in [18, [19 and 20]. Unlike such approaches, this paper tries to find a compromise between an above sketched multidisciplinary group of problems and the need to design and implement rather simple computations. Coming out from the principle of energy conservation from classical mechanics, incorporating the kinetic and deformation energy, together with certain energy dissipation (structural and mass damping), the initiation of some micro-cracks will be incorporated using the approach of [21] and [9], utilizing numerous ideas of 22, adopting the nonlocal model from 23]. This model was later reformulated by 24 and is frequently referred as the Eringen one in the last 2 decades; for more motivations and references to nonlocal models in continuum mechanics cf. 25]. For the strain-stress relations we shall start with the linearized viscoelastic Kelvin law. Such a model problem will be discussed in Section II. in details. Available generalizations, handling the quasi-static case, discussed in 26] separately, as well as composite structures and cohesive interfaces, motivated by [27, 4], 28], 29] (for various types of fibre composites), 30 (for ceramics) and 31] (for a rather general class of damage propagation), will be sketched in Section III. Section IV. shows the possibility of development of relevant software, including several numerical examples. Section V. contains some ideas for more complex formulations, not covered by Sections II. and III. properly, involving open problems for future research.
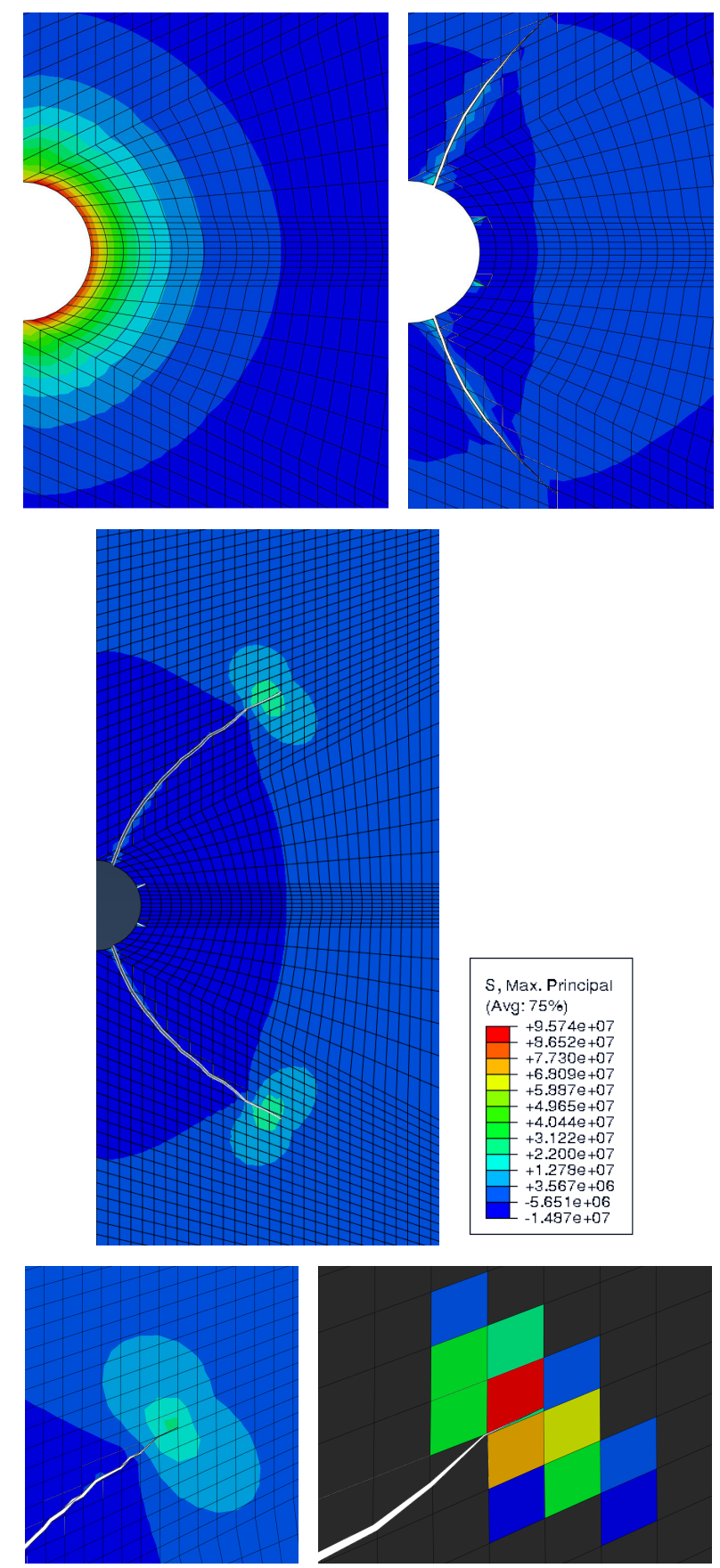

Fig. 1: Creation and propagation of a crack using XFEM in a cement paste without any stiffening fibres. 


\section{A MODEL PROBLEM}

Due to the limited extent of this conference paper, to save its reader-friendliness, we shall start with the formulation of a rather simple model problem on a (at least macroscopically) one-component material, whose solvability, convergence of approximate solutions derived from Rothe sequences, etc., could be handled by standard arguments on linear parabolic and hyperbolic equations of evolution by [32], with just one exception: stiffness weakening due to certain damage factor, derived from the nonlocal stress evaluation. More complicated both geometrical and physical configurations will be mentioned in Section III. and implemented in Section IV.

\section{A. Mathematical preliminaries}

Let us consider a domain $\Omega$ in the 3 -dimensional Euclidean space $\mathcal{R}^{3}$, whose exterior Lipschitz boundary $\partial \Omega$ consists of 2 disjoint parts $\Theta$ (for homogeneous Dirichlet boundary conditions) and $\Gamma$ (for nonhomogeneous Neumann boundary conditions), $\Theta$ having a non-zero measure on $\partial \Omega$ (to avoid insufficient support). Let $\mathcal{R}^{3}$ be supplied by a Cartesian coordinate system $x=\left(x_{1}, x_{2}, x_{3}\right)$. Moreover we shall work with the time $t \in I, I=[0, T]$ for some final time value $T$, assumed as finite here. For the brevity of notation we shall work with the Hamilton operator $\nabla=\left(\partial / \partial x_{1}, \partial / \partial x_{2}, \partial / \partial x_{3}\right)$, with upper dots instead of $\partial / \partial t$. Moreover any comma followed by $k \in\{1,2,3\}$ will be seen as $\partial / \partial x_{k}$ applied to the preceding variable: e.g. $2 \varepsilon_{i j}(v)=v_{i, j}+v_{j, i}$ with $i, j \in\{1,2,3\}$ can be understood as the definition of linearized strain, applicable to any differentiable virtual displacement $v(x)=\left(v_{1}(x), v_{2}(x), v_{3}(x)\right)$, related to an initial configuration. The Einstein summation rule for indices $i, j, k, l \in\{1,2,3\}$ will be active, too.

The introduction of Lebesgue, Sobolev and Bochner spaces of functions on $\Omega$ and $\partial \Omega$ and abstract functions mapping $I$ to them is compatible with 33. Namely we shall work with the Hilbert spaces $H=L^{2}(\Omega)^{3}$, $Z=L^{2}(\partial \Omega)^{3}, Z_{\Gamma}=L^{2}(\Gamma)^{3}$ and $V=\left\{v \in W^{1,2}(\Omega)^{3}\right.$ : $v=\emptyset$ on $\Theta\}$, supplied with norms $|$.$| both in H$ and $H \times H,|\cdot|_{\Gamma}$ in $Z_{\Gamma}$ and $\|\cdot\|$ in $V$, as well as with scalar products $(.,$.$) both in H$ and $H \times H$, together with $\langle., .\rangle_{\Gamma}$ in $Z_{\Gamma}$. We shall also utilize upper star symbols for dual spaces, $\subset$ for continuous embeddings, $\Subset$ for compact embeddings, $\cong$ for identification of a space with its dual in the sense of the Riesz representation theorem.

The following properties of the above introduced spaces (for all notations and proofs see 33 again) will be needed:

P1) The Bochner - Sobolev space $L^{2}(I, V)^{*} \cong L^{2}\left(I, V^{*}\right)$ is reflexive; consequently from any bounded sequence in $L^{2}(I, V)$ a weakly convergent subsequence can be selected (the Eberlein-Shmulyan theorem).

P2) $H \Subset V$ (the Sobolev embedding theorem).

P3) $Z \Subset V$; thus $|v|_{\Gamma}^{2} \leq \mathfrak{T}\|v\|^{2}$ for any $v \in V$ with a positive $\mathfrak{T}$ independent of $v$ (the trace theorem).
P4) In addition to the standard norm $\|v\|^{2}=|v|^{2}+|\nabla v|^{2}$ for any $v \in V$, an alternative norm in $V$ is generated by $|\varepsilon(v)|^{2}$ because $|\varepsilon(v)|^{2} \leq|\nabla v|^{2} \leq\|v\|^{2}$ and $|\varepsilon(v)|^{2} \geq \mathfrak{K}\|v\|^{2}$ with a positive $\mathfrak{K}$ independent of $v$ (the Korn inequality).
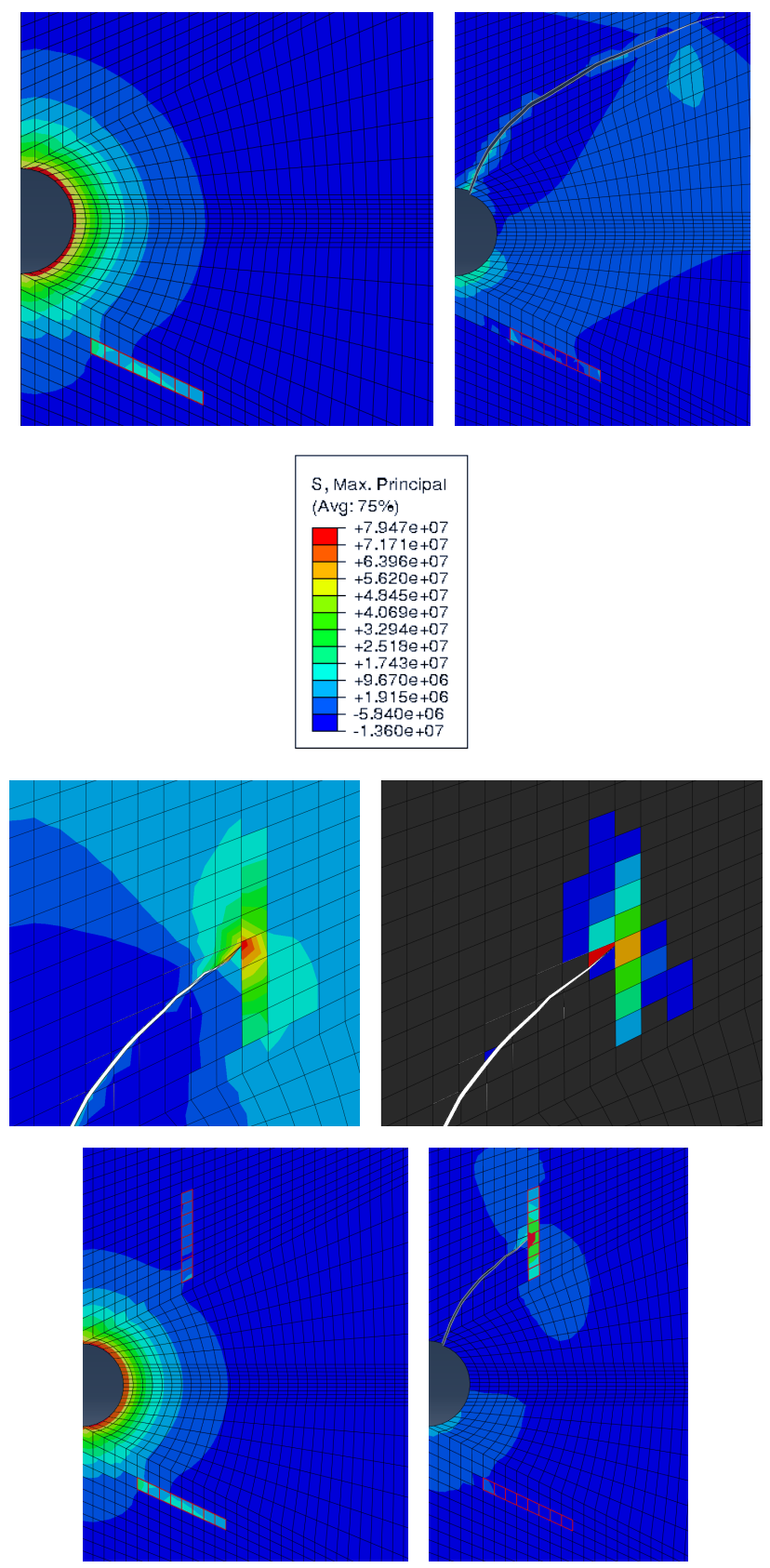

Fig. 2: Results comparable with Fig. 1 for 1 and 2 stiffening fibres.

\section{B. Dynamics of a viscoelastic body}

Let us introduce an displacement in a deformable body (a priori uknown), occupying the domain $\Omega$, $u(x, t)=\left(u_{1}(x, t), u_{2}(x, t), u_{3}(x, t)\right)$, related to the reference initial configuration (at $t=0)$, such that the homogeneous Cauchy initial conditions $u_{1}(x, 0)=u_{2}(x, 0)=$ $u_{3}(x, 0)=0$ and $\dot{u}_{1}(x, 0)=\dot{u}_{2}(x, 0)=\dot{u}_{3}(x, 0)=0$ are satisfied for almost every $x \in \Omega$. We shall assume that 
$u \in W^{1,2,2}(I, V, V)$; both initial conditions can be written as

$$
u(., 0)=\emptyset, \quad \dot{u}(., 0)=\emptyset \text { on } \Omega .
$$

Analogous simplified notations will be used for further functions, too.

For an arbitrary $v \in V$ the energy conservation for our model problem can be presented in its weak (integral) formulation

$$
\begin{aligned}
& (v, \rho \dot{u})+\beta(v, \rho u)+\alpha(\varepsilon(v), \sigma) \\
& +[(\varepsilon(v),(1-\mathfrak{D}) \sigma)]=[(v, f)]+\left[\langle v, g\rangle_{\Gamma}\right] \text { on } I
\end{aligned}
$$

where

$$
[\phi, \psi](t)=\int_{0}^{t} \phi(\xi) \psi(\xi) \mathrm{d} \xi
$$

is introduced for arbitrary functions $\phi, \psi \in L^{2}(I)$ over $[0, t]$ ( $t$ in 2 and later is not emphasized explicitly for brevity). Here $\rho \in L^{\infty}(\Omega)$ is the material density and $\sigma \in L^{2}\left(I, H_{\text {sym }}^{3 \times 3}\right)$ refers to all stress components. Its symmetry comes from the assumptions on Boltzmann continuum; for much more general considerations of this type, including constitutive laws, cf. [34, p. 18, and 35]. The energy dissipation in (2), driven by the prescribed body forces $f \in L^{2}\left(I, H^{3}\right)$ and surface forces $g \in L^{2}\left(I, Z_{\Gamma}^{3}\right)$, is taken into account using the positive damping factors $\alpha$ for structural damping due to the parallel Kelvin viscoelastic model, and the real non-negative factor $\beta$ for mass damping, compatible with the Rayleigh damping model by 36. We shall assume that $\rho \geq \rho_{0}$ on $\Omega$ for some positive constant $\rho_{0}$. Finally $\mathfrak{D}$ can be presented as some damage factor with values between 0 , assumed always for $t=0$, and $1-\varsigma$, using an additional positive constant $\varsigma$.

Let us remind that the strong (differential) formulation corresponding to 2 can be derived, at least in the sense of distributions, from integration of by parts. Following the approach of [26] (where the quasi-static case is discussed in all details), for each $i$ we receive

$$
\begin{array}{rll}
\rho\left(\ddot{u}_{i}+\beta \dot{u}_{i}\right) & & \\
-\left(\alpha \dot{\sigma}_{i j}+(1-\mathfrak{D}) \sigma_{i j}\right)_{, j}=f_{i} & \text { on } & \Omega \times I, \\
\left(\alpha \dot{\sigma}_{i j}+(1-\mathfrak{D}) \sigma_{i j}\right) \nu_{j}=g_{i} & \text { on } & \Gamma \times I, \\
u_{i}=\emptyset & \text { on } & \Theta \times I
\end{array}
$$

where $\nu=\left(\nu_{1}, \nu_{2}, \nu_{3}\right)$ means the local unit normal vector associated with $\Gamma$. In addition to the 1 st evolution equation of (3), referring to the classical Cauchy equilibrium condition, we can see both an explicit Neumann boundary condition in the 2 nd equation and a Dirichlet one in the 3rd equation.

The local stress-strain relation can be taken in the simple form

$$
\sigma=C \varepsilon(u)
$$

with $C \in L^{\infty}(\Omega)_{\mathrm{sym}}^{(3 \times 3) \times(3 \times 3)}$, containing (in general) 21 material parameters, $C(x)$ being positive definite in the sense $C_{i j k l}(x) a_{i j} a_{k l} \geq C_{0} a_{i j} a_{i j}$, involving some positive constant $C_{0}$. In particular, for an isotropic homogeneous medium, using the Kronecker symbol $\delta_{i j}=1$ for $i=j$, 0 otherwise, we have

$$
\sigma_{i j}=2 \lambda_{1} \varepsilon_{i j}(u)+\lambda_{2} \delta_{i j} \varepsilon_{k k}(u)
$$

with only 2 positive Lamé factors $\lambda_{1}$ and $\lambda_{2}$; frequently they are expressed as

$$
\lambda_{1}=\mu E /(1+\mu) /(1-2 \mu), \quad 2 \lambda_{2}=E /(1+\mu),
$$

utilizing the well-known Young modulus $E$ and the Poisson ratio $\mu$ (cf. Section IV.).

\section{Nonlocal damage factor}

Our final aim is to derive $u \in W^{1,2,2}(I, V, V)$ from (2). Since $\sigma$ can be inserted from (4) into (2), the remaining step is to express $\mathfrak{D}$ as an appropriate function of $\sigma$, with certain regularizing properties. This can be done using some kernel (typically radial basis or similar) operator $\mathcal{K} \in L^{2}(\Omega \times \Omega)$, introduced as

$$
A(w(x))=\int_{\Omega} K(x, \tilde{x}) w(\tilde{x}) \mathrm{d} \tilde{x}
$$

for $x \in \Omega$ and $w \in H$ by 24]. The needed generalization for $w \in H_{\mathrm{sym}}^{3 \times 3}$, as introduced by 37, or that for effective mean stresses (for the sake of objectivity) by [38, is straightforward. Namely 37] works with $K(x, \tilde{x})=\mathcal{K}\left(|x-\tilde{x}|_{3}\right)$ where $|\cdot|_{3}$ means the norm in $\mathcal{R}^{3}$ and $\mathcal{K}\left(|x-\tilde{x}|_{3}\right)$ is obtained using Green functions of the bi-Helmholtz equation

$$
\left(1-c_{1}^{2} \Delta\right)\left(1-c_{2}^{2} \Delta\right) \mathcal{K}\left(|x-\tilde{x}|_{3}\right)=\delta(x-\tilde{x}) ;
$$

$\delta$ here refers to the Dirac distribution and $\Delta=\partial^{2} / \partial x_{1}^{2}+$ $\partial^{2} / \partial x_{2}^{2}+\partial^{2} / \partial x_{3}^{2}$ to the Laplace operator. Thus

$$
\begin{aligned}
\mathcal{K}(\xi)=\frac{1}{4 \pi} \cdot \frac{1}{c_{1}^{2}-c_{2}^{2}} \cdot \frac{1}{\xi} & \left(\exp \left(-|\xi|_{3} / c_{1}\right)\right. \\
- & \left.\exp \left(-|\xi|_{3} / c_{2}\right)\right)
\end{aligned}
$$

for a positive $\xi$ and

$$
\mathcal{K}(0)=\frac{1}{4 \pi} \cdot \frac{1}{c_{1} c_{2}\left(c_{1}+c_{2}\right)},
$$

assuming $c_{1} \neq c_{2}$. Here $c_{1}$ and $c_{2}$ are 2 material length scales, which can be traced up to atomistic considerations, working with dislocation and disclination defects. Limit cases as $c_{1} \rightarrow c_{2}$ and $c_{2} \rightarrow 0$ (referring to the standard Helmholtz equations) can be adopted easily. However, for more complicated material structures such transparent theory is not available; e.g. for practical computational simulations of behaviour of fibrereinforced concrete structures under mechanical loads 39 recommends the "generalized Mazars model" with several heuristic parameters, respecting anisotropy together with different behaviour under tension and pressure like [9] and [40], inspired by [41], [42] and [21].

Fortunately the recent result 43 on the ill-possedness of the nonlocal approach 24], referring to the existence analysis [4], for boundary conditions significant in practical applications is not addressed to our formulation, as 
explained in 26$]$. Therefore we are ready to work with $\tilde{\sigma}=A(\sigma)$ with values from $\mathcal{R}_{\text {sym }}^{3 \times 3}$ (or its natural modification, as mentioned above) and to compute

$$
\mathfrak{D}=\max _{\xi \in[0, t]} \omega(\tilde{\sigma}(\xi))
$$

where $\omega$ is some real continuous non-decreasing function (containing additional experimentally validated parameters typically) defined on $\mathcal{R}_{\text {sym }}^{3 \times 3}$ with all values lesser of equal to $1-\varsigma$; the maximization forces the irreversibility of damage. Clearly $\mathfrak{D}$ depends on $u$ from (2) (in a rather complicated way), which will not be highlighted in the rest of this paper formally. Nevertheless, the definition (6) together with (5) enables us to exploit the results on Nemytyskiî mappings by [45], p. 134: if some sequence converges weakly to $u \in V$ for a fixed $t \in I$, thanks to (4), transforms it to a weakly convergent sequence to $\sigma \in H$, after the regularization $\tilde{\sigma}=A(\sigma)$ the same, up to a subsequence, converges strongly to $\tilde{\sigma} \in H$, etc. Consequently, thanks to the continuity of $\omega$, we are allowed to come to the strong limit of the corresponding sequence induced by (6), which may be helpful to overcome the nonlinearity of our model problem. However, the design of a sufficiently general class of functions $\omega$ admitting all above sketched mathematical considerations and applicable in engineering practice (regardless of both physical and geometrical linearizations, together with the existence of a positive $\varsigma$ ) cannot be seen as a closed problem; for some particular examples cf. 9 and $[26$.
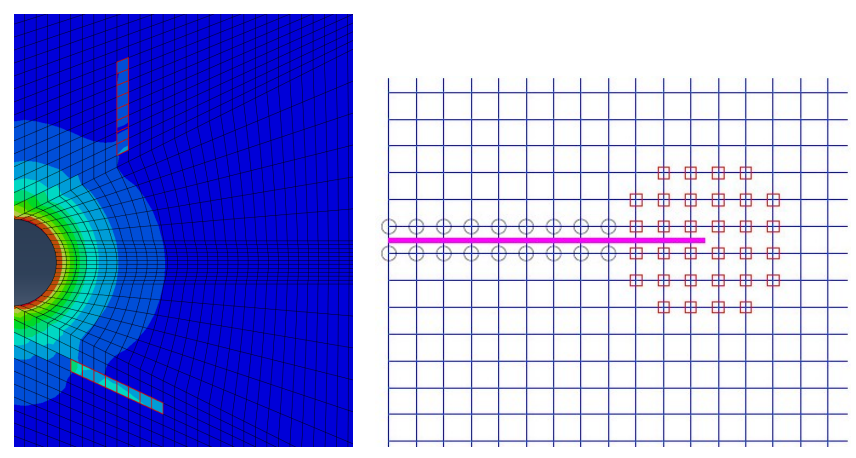

Fig. 3: Effect of nonlocal stress evaluation at the crack tip.

\section{Convergence of Rothe sequences}

The computational algorithm for the analysis of (2), with inserted $\sigma$ from (4), assuming $\mathfrak{D}$ (also dependent of $u$ ) evaluated from $(6)$, i. e.

$$
\begin{aligned}
& (v, \rho \dot{u})+\beta(v, \rho u) \\
& +\alpha(\varepsilon(v), C \varepsilon(u)) \\
& +[(\varepsilon(v),(1-\mathfrak{D}) C \varepsilon(u))] \\
& =[(v, f)]+\left[\langle v, g\rangle_{\Gamma}\right] \text { on } I,
\end{aligned}
$$

will rely on the evaluation of 3 special types of Rothe sequences. Let $I$ be divided into a finite number $m$ of subsets

$$
I_{s}^{m}=\{t \in I:(s-1) \tau<t \leq s \tau\}, \quad s \in\{1, \ldots, m\},
$$

with the final aim $m \rightarrow \infty ; \tau(m)=T / m$ is considered (the argument $m$ will be omitted formally). We are able to work with the Clément quasi-interpolation $f^{m}$ of $f$ in $L^{2}(I, H)$ and $g^{m}$ of $g$ in $L^{2}\left(I, Z_{\Lambda}\right)$, assuming $t \in I_{s}^{m}$, $s \in\{1, \ldots, m\}$, i. e.

$$
\begin{aligned}
f^{m}(t) & =\frac{1}{\tau} \int_{(s-1) \tau}^{s \tau} f(\xi) \mathrm{d} \xi, \\
g^{m}(t) & =\frac{1}{\tau} \int_{(s-1) \tau}^{s \tau} g(\xi) \mathrm{d} \xi .
\end{aligned}
$$

For any unknown $u^{m}$ we have to set some linear Lagrange splines

$$
u^{m}(t)=u_{s-1}^{m}+(t-(s-1) \tau)\left(u_{s}^{m}-u_{s-1}^{m}\right)
$$

and standard and retarded simple functions

$$
\bar{u}^{m}(t)=u_{s}^{m}, \quad \breve{u}^{m}(t)=u_{s-1}^{m},
$$

taking $u_{0}^{m}=\emptyset$. Later we shall nead also the 1 st and 2 nd differences $\mathcal{D} u_{s}^{m}=u_{s}^{m}-u_{s-1}^{m}$ and $\mathcal{D}^{2} u_{s}^{m}=\mathcal{D} u_{s}^{m}-\mathcal{D} u_{s-1}^{m}$ with $s=\{1, \ldots, m)$, taking $u_{0}^{m}=\emptyset$ and $\mathcal{D} u_{0}^{m}=\emptyset$.

The discrete variant of (7) reads

$$
\begin{aligned}
& \left(v, \rho \dot{u}^{m}\right)+\beta\left(v, \rho \bar{u}^{m}\right) \\
& +\alpha\left(\varepsilon(v), C \varepsilon\left(\bar{u}^{m}\right)\right) \\
& +\left[\left(\varepsilon(v),\left(1-\breve{\mathfrak{D}}^{m}\right) C \varepsilon\left(\bar{u}^{m}\right)\right)\right] \\
& =\left[\left(v, f^{m}\right)\right]+\left[\left\langle v, g^{m}\right\rangle_{\Gamma}\right] \text { on } I_{s}^{m} ;
\end{aligned}
$$

here $\breve{\mathfrak{D}}^{m}$ refers to $\mathfrak{D}$ calculated for $\breve{u}^{m}$ instead of $u$. In terms of the values $u_{1}^{m}, \ldots, u_{m}^{m}$, taking $r \in\{1, \ldots, s\}$ as the Einstein summation index (and $\delta_{r r}$ as the 'ecker symbol formally) for each $s \in\{1, \ldots, m\}$ (7) gives

$$
\begin{aligned}
& \frac{1}{h}\left(v, \rho \mathcal{D} u_{s}^{m}\right)+\beta\left(v, \rho u_{s}^{m}\right) \\
& +\left(\varepsilon(v), \alpha C \varepsilon\left(u_{s}^{m}\right)\right) \\
& +h \delta_{r r}\left(\varepsilon(v),\left(1-\mathfrak{D}_{r-1}^{m}\right) C \varepsilon\left(u_{r}^{m}\right)\right) \\
& =h \delta_{r r}\left(v, f_{r}^{m}\right)+h \delta_{r r}\left\langle v, g_{r}^{m}\right\rangle_{\Gamma} ;
\end{aligned}
$$

here $\mathfrak{D}_{s-1}^{m}$ refers to $\mathfrak{D}$ calculated for $u_{s-1}^{m}$ instead of $u$, too. Subtracting $s$-th and $(s-1)$-th equations (11), we obtain

$$
\begin{aligned}
& \frac{1}{h}\left(v, \rho \mathcal{D}^{2} u_{s}^{m}\right)+\beta\left(v, \rho \mathcal{D} u_{s}^{m}\right) \\
& +\alpha\left(\varepsilon(v), C \varepsilon\left(\mathcal{D} u_{s}^{m}\right)\right) \\
& +h\left(\varepsilon(v),\left(1-\mathfrak{D}_{s-1}^{m}\right) C \varepsilon\left(u_{s}^{m}\right)\right) \\
& =h\left(v, f_{s}^{m}\right)+h\left\langle v, g_{s}^{m}\right\rangle_{\Gamma},
\end{aligned}
$$

which is the desired formula for the step-by-step evaluation of $u_{s}^{m}$.

Now we shall exploit 12 to derive some a priori bounds for all sequences $\left\{u^{m}\right\}_{m=1}^{\infty},\left\{\dot{u}^{m}\right\}_{m=1}^{\infty},\left\{\bar{u}^{m}\right\}_{m=1}^{\infty}$ and $\left\{\breve{u}^{m}\right\}_{m=1}^{\infty}$. Choosing $v=\mathcal{D} u_{s}^{m} / h$ in 12 , we have

$$
\begin{aligned}
& \frac{1}{h^{2}}\left(\mathcal{D} u_{s}^{m}, \rho \mathcal{D}^{2} u_{s}^{m}\right)+\frac{\beta}{h}\left(\mathcal{D} u_{s}^{m}, \rho \mathcal{D}^{2} u_{s}^{m}\right) \\
& +\frac{\alpha}{h}\left(\varepsilon\left(\mathcal{D} u_{s}^{m}\right), C \varepsilon\left(\mathcal{D} u_{s}^{m}\right)\right) \\
& +\left(\varepsilon\left(\mathcal{D} u_{s}\right),\left(1-\mathfrak{D}_{s-1}^{m}\right) C \varepsilon\left(u_{s}\right)\right) \\
& =\left(\mathcal{D} u_{s}, f_{s}^{m}\right)+\left\langle\mathcal{D} u_{s}, g_{s}^{m}\right\rangle_{\Gamma}
\end{aligned}
$$


The same results remains true with arbitrary $r \in$ $\{1, \ldots, s\}$ instead of $s$. Using the obvious relation $2 a(a-b)=a^{2}-b^{2}+(a-b)^{2}$, valid for any real $a$ and $b$, the sum of all such equations derived from 13 is then

$$
\begin{aligned}
& \frac{1}{2 h^{2}}\left(\mathcal{D} u_{s}^{m}, \rho \mathcal{D} u_{s}^{m}\right) \\
& +\frac{1}{2 h^{2}} \delta_{r r}\left(\mathcal{D}^{2} u_{r}^{m}, \rho \mathcal{D}^{2} u_{r}^{m}\right) \\
& +\frac{\beta}{h}\left(\mathcal{D} u_{r}^{m}, \rho \mathcal{D} u_{r}^{m}\right)+\frac{\alpha}{h}\left(\varepsilon\left(\mathcal{D} u_{r}^{m}\right), C \varepsilon\left(\mathcal{D} u_{r}^{m}\right)\right) \\
& +\frac{1}{2}\left(\varepsilon\left(u_{s}^{m}\right),\left(1-\mathfrak{D}_{s}^{m}\right) C \varepsilon\left(u_{s}^{m}\right)\right) \\
& +\frac{1}{2}\left(\varepsilon\left(u_{r}^{m}\right),\left(\mathfrak{D}_{r}^{m}-\mathfrak{D}_{r-1}^{m}\right) \varepsilon\left(u_{r}^{m}\right)\right) \\
& +\frac{1}{2}\left(\varepsilon\left(\mathcal{D} u_{r}^{m}\right),\left(1-\mathfrak{D}_{r-1}^{m}\right) C \varepsilon\left(\mathcal{D} u_{r}^{m}\right)\right) \\
& =\left(\mathcal{D} u_{r}^{m}, f_{r}^{m}\right)+\left\langle\mathcal{D} u_{r}^{m}, g_{r}^{m}\right\rangle_{\Gamma} .
\end{aligned}
$$

All left-hand-side additive terms are non-negative, thus the 2nd, 3rd, 6th (thanks to the careful introduction of (6) and 7th ones can be seen as bounded from below by zero, whereas the 1st, 3rd and 5th ones together with P4) (see Part A above) admit the more careful estimates

$$
\begin{aligned}
\frac{1}{2 h^{2}}\left(\mathcal{D} u_{s}^{m}, \rho \mathcal{D} u_{s}^{m}\right) & \geq \frac{\rho_{0}}{2 h^{2}}\left|\mathcal{D} u_{s}^{m}\right|^{2}, \\
\frac{\alpha}{h}\left(\varepsilon\left(\mathcal{D} u_{r}^{m}\right), \alpha C \varepsilon\left(\mathcal{D} u_{r}^{m}\right)\right) & \geq \frac{\alpha C_{0} \mathfrak{K}}{h} \delta_{r r}\left\|\mathcal{D} u_{r}^{m}\right\|^{2}, \\
\frac{1}{2}\left(\varepsilon\left(u_{s}^{m}\right),\left(1-\mathfrak{D}_{s}^{m}\right) C \varepsilon\left(u_{s}^{m}\right)\right) & \geq \frac{\varsigma C_{0} \mathfrak{K}}{2}\left\|u_{s}^{m}\right\|^{2} .
\end{aligned}
$$

Using the Cauchy-Schwarz and the Young inequalities, the 1st and 2nd right-hand-side terms can be estimated with help of P3) as

$$
\begin{aligned}
\left(\mathcal{D} u_{r}^{m}, f_{r}^{m}\right) & \leq\left|\mathcal{D} u_{r}^{m}\right|\left|f_{r}^{m}\right| \\
& \leq \frac{\epsilon}{2 h} \delta_{r r}\left|\mathcal{D} u_{r}^{m}\right|^{2}+\frac{h}{2 \epsilon} \delta_{r r}\left|f_{r}^{m}\right|^{2} \\
& \leq \frac{\epsilon}{2 h} \delta_{r r}\left\|\mathcal{D} u_{r}^{m}\right\|^{2}+\frac{h}{2 \epsilon} \delta_{r r}\left|f_{r}^{m}\right|^{2}, \\
\left\langle\mathcal{D} u_{r}^{m}, g_{r}^{m}\right\rangle_{\Gamma} & \leq\left|\mathcal{D} u_{r}^{m}\right|_{\Gamma}\left|g_{r}^{m}\right|_{\Gamma} \\
& \leq \frac{\epsilon}{2 h} \delta_{r r}\left|\mathcal{D} u_{r}^{m}\right|_{\Gamma}^{2}+\frac{h}{2 \epsilon} \delta_{r r}\left|g_{r}^{m}\right|_{\Gamma}^{2} \\
& \leq \frac{\epsilon \mathfrak{T}}{2 h} \delta_{r r}\left\|\mathcal{D} u_{r}^{m}\right\|^{2}+\frac{h}{2 \epsilon} \delta_{r r}\left|g_{r}^{m}\right|_{\Gamma}^{2},
\end{aligned}
$$

where $\epsilon$ is an arbitrary positive constant. From (14, , 16 and 15$]$ we can conclude

$$
\frac{1}{h^{2}}\left|\mathcal{D} u_{s}\right|^{2}+\frac{1}{h} \delta_{r r}\left\|\mathcal{D} u_{r}\right\|^{2}+\left\|u_{s}\right\|^{2} \leq c
$$

with some positive constant $c$ independent of $h$ (as well as of $m, s$, etc.). This guarantees the following boundedness for the Rothe sequences:

$\left\{\dot{u}^{m}\right\}_{m=1}^{\infty}$ is bounded in $L^{2}(I, V)$, $\left\{\dot{u}^{m}(t)\right\}_{m=1}^{\infty}$ is bounded in $H$ for any $t \in I$, $\left\{\bar{u}^{m}(t)\right\}_{m=1}^{\infty}$ is bounded in $V$ for any $t \in I$, $\left\{\breve{u}^{m}(t)\right\}_{m=1}^{\infty}$ is bounded in $V$ for any $t \in I$.
Consequently P1) yields, up to subsequences, using $\rightarrow$ instead of "converges strongly to" and $\rightarrow$ for "converges weakly to" for brevity,

$$
\begin{gathered}
\left\{\dot{u}^{m}\right\}_{m=1}^{\infty} \rightarrow \hat{u} \text { in } L^{2}(I, V), \\
\left\{\dot{u}^{m}(t)\right\}_{m=1}^{\infty} \rightarrow u^{\prime} \text { in } H \text { for any } t \in I, \\
\left\{\bar{u}^{m}(t)\right\}_{m=1}^{\infty} \rightarrow \bar{u} \text { in } V \text { for any } t \in I, \\
\left\{\breve{u}^{m}(t)\right\}_{m=1}^{\infty} \rightarrow \breve{u} \text { in } V \text { for any } t \in I, \\
\left\{\dot{u}^{m}\right\}_{m=1}^{\infty} \rightarrow \hat{u} \text { in } L^{2}(I, H), \\
\left\{\breve{u}^{m}(t)\right\}_{m=1}^{\infty} \rightarrow \breve{u} \text { in } H \text { for any } t \in I,
\end{gathered}
$$

etc., where $u^{\prime}, \bar{u}, \breve{u}$ and $\hat{u}$ are some elements of corresponding spaces; the 5 th and 6 th proposition (18) (and similar ones) need also P2). The strong convergence of $\breve{\mathfrak{D}}^{m}$ occurring in 10 , corresponding to the 6 th proposition, must then rely (6), as discussed in Part C. The 1st and 2 nd propositions manifest the weak convergence of the same sequence both to $\hat{u}$ and to $u^{\prime}$ in e.g. in $L^{2}(I, H)$, thus $\hat{u}=u^{\prime}$. The estimate

$$
\begin{aligned}
& \max \left(\left|u^{m}(t)-\bar{u}^{m}(t)\right|,\left|u^{m}(t)-\breve{u}^{m}(t)\right|\right) \\
& \leq \max _{s \in\{1, \ldots, m\}}\left|\mathcal{D} u_{s}\right| \leq \sqrt{c} h=\frac{\sqrt{c} T}{m}
\end{aligned}
$$

implies $u=\bar{u}=\breve{u}$ and $\dot{u}=u^{\prime}$ where

$$
u(t)=\int_{0}^{t} u^{\prime}(\xi) \mathrm{d} \xi
$$

for any $t \in I$. This guarantees the possibility of limit passage with $m \rightarrow \infty$ from 10 to 2 .

\section{AVAILABle GENERALIZATIONS}

Some generalizations of Section II. seem to be straightforward, but bring non-negligible technical difficulties. Namely $H$ in P2) could be replaced by some $L^{p}(\Omega)^{3}$ with $p=6-\epsilon$ where $0<\epsilon \leq 4$, as well as $Z_{\Gamma}$ in P3) by $L^{q}(\Gamma)^{3}$ with $q=4-\tilde{\epsilon}$ where $0<\tilde{\epsilon} \leq 2$; therefore the assumptions $f \in L^{2}\left(I, L^{p /(p-1)}(\Omega)\right)$ and $g \in L^{2}\left(I, L^{q /(q-1)}(\Gamma)\right)$ could be sufficient in (7), instead of those with $\epsilon=4$ and $\tilde{\epsilon}=2$, which gives $p=q=2$ ). Even the assumption on Lipschitz boundary can be weakened, as discussed by [46], 47] and [48]. However, such spaces are not Hilbert ones, simple analytical tools like the Riesz representation theorem are not available, scalar products must be substituted by more general dualities, the less transparent convergence analysis in dual spaces is required, etc. As the first step, useful in some following applications, the following propositions can be added to P1), P2), P3) and P4):

P5) In the Gelfand triple $V \subset H \cong H^{*} \subset V^{*}$ both inclusions are dense; $W^{1,2,2}\left(I, V, V^{*}\right) \subset C(I, H)$.

P6) $W^{1,2,2}\left(I, V, V^{*}\right) \Subset L^{2}(I, X)$ with $X \in\{H, Z\}$ (the Aubin-Lions lemma).

\section{A. Macroscopic cracks}

Instead of one domain $\Omega$, as introduced by Section II. we can consider a union of a finit number of adjacent 
domains, denoted by $\Omega$ again, whose boundary $\partial \Omega$ consists of 3 parts: of 2 exterior ones, analogical to $\Gamma$ and $\Theta$, and of a set of internal interfaces $\Lambda$. Such notion of interfaces can cover both potential locations of macrocracks, as well as existing interfaces between particular components of a composite, e.g. between a cementitious matrix and stiffening fibres in building applications.
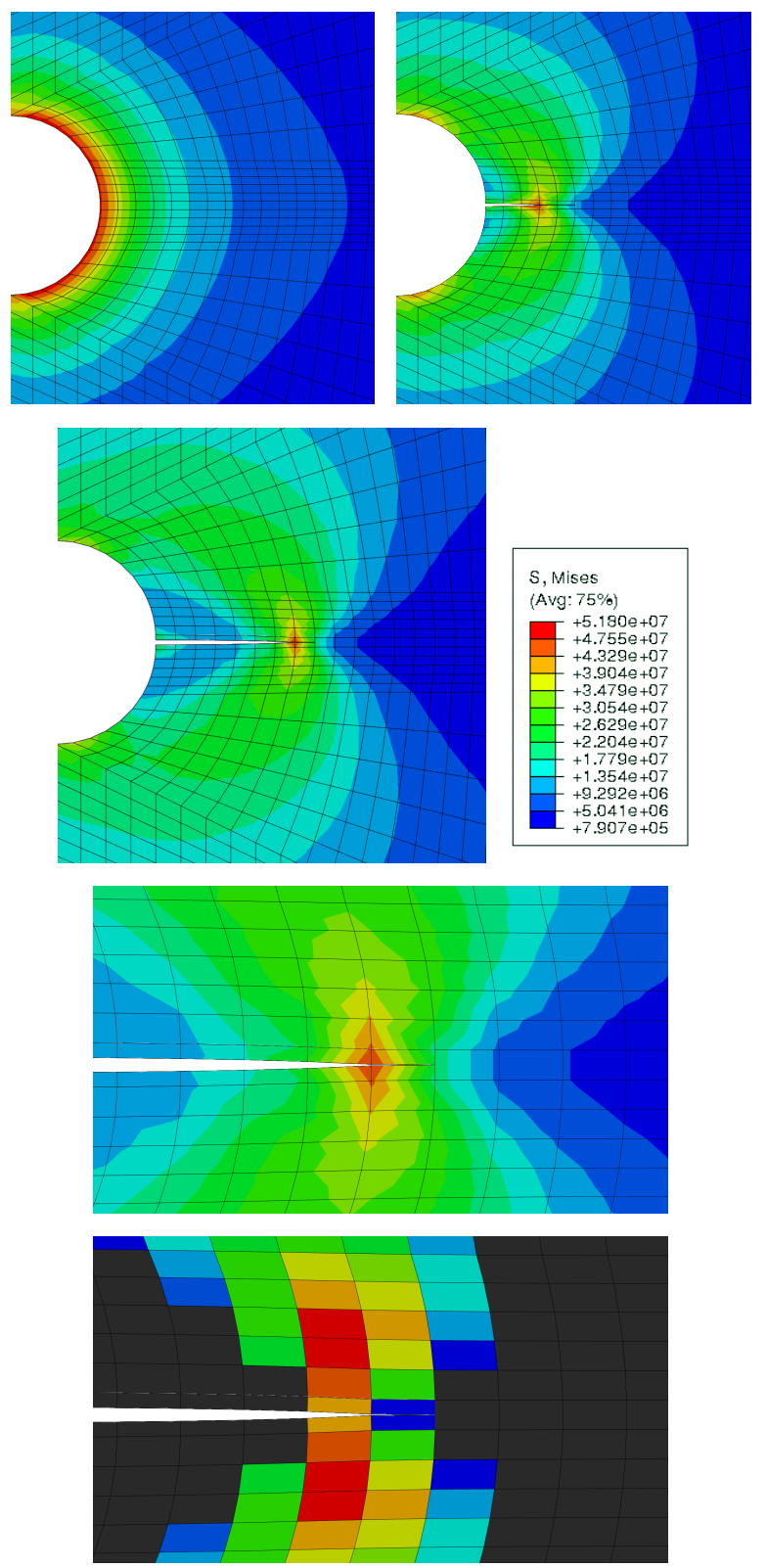

Fig. 4: Nonlocal damage model Mises stress with the evaluation of damage factor by [21], applying the exponential formula from [41], close to 2 fibres.

The contacts on $\Lambda$ could be seemingly handled similarly to the surface loads on $\Gamma$, except their a priori knowledge, but such approach is not realistic. The behaviour of $\Lambda$ must be seen as result of mutual interaction of parts of $\Omega$, which requires some deeper physical analysis. However, we can introduce the space $Z_{\Lambda}$, the norm $|.|_{\Lambda}$ and the scalar product $\langle., .\rangle_{\Lambda}$ quite analogically to $Z_{\Gamma},|.|_{\Gamma}$ and $\langle., .\rangle_{\Gamma}$. Such approach offers a possibility of coupling micro-fracture (using the damage factor) with opening and propagation of macroscopic cracks.

\section{B. Cohesive interfaces}

Quantification of the phenomena connected with $\Lambda$ can be performed utilizing the cohesive model by 29$]$ and 31. Opening and closing of interfaces can be expressed using the relation

$$
\mathfrak{T}=\lambda(\delta u) \text { on } \Lambda \times I,
$$

as certain analogue of (4); $\delta u$ here refer to the jumps in values of $u$ on $\Lambda$, the same notation will be applied to $v$, $\mathfrak{T}$ denotes the related contact stress. Appropriate forms of a just introduced function $\lambda$ can be found in $[28$ and 30. Consequently $\left[\langle\delta v, \tau\rangle_{\Gamma}\right]$ is allowed to be joined as an additional right-hand-side term to (2), with the obvious modification of (3), too. In particular, $\lambda(\delta u)=\lambda_{0} \delta u$ with a real constant $\lambda_{0} \rightarrow \infty$ forces $\delta u \rightarrow \emptyset$ on $\Lambda$, i. e. the continuity of $u$ without no active macro-cracking. We can see that 20 brings a new type of nonlinearity to our problem in all other cases.

Let us suppose that $\lambda$ is a Lipschitz continuous mapping from $Z_{\Lambda}$ to $Z_{\Lambda}$. For some positive constant $\lambda_{\star}$ this yields

$$
|\lambda(\delta v)|_{\Lambda}^{2} \leq \lambda_{\star}|\delta v|_{\Lambda}^{2} \leq \lambda_{\star} \mathfrak{L}\|v\|^{2}
$$

for any $v \in V ; \mathfrak{L}$ here can be seen as an analogy of $\mathfrak{T}$ in $\mathrm{P} 1$ ) in Section II. multiplied by certain factor depending on geometrical configuration, interpretable as the maximal number of parts of $\Lambda$ creating an interface of one fixed part of $\Omega$.

Following the approach of Section II. completely, we come to the trailing additional term $\left\langle\overline{\delta \mathcal{D}} u_{r}^{m}, \lambda\left(\delta u_{r-1}^{m}\right)\right\rangle_{\Lambda}$ of (14); a seemingly better choice $\delta u_{r}^{m}$ instead of $\delta u_{r-1}^{m}$ here would force nonlinear algebraic computations. However, this can be overcome using the estimate

$$
\begin{aligned}
& \left\langle\delta \mathcal{D} u_{r}, \lambda\left(\delta u_{r-1}\right)\right\rangle_{\Lambda} \leq \lambda_{\star}\left|\delta \mathcal{D} u_{r}\right|_{\Lambda}\left|\delta u_{r-1}\right|_{\Lambda} \\
& \leq \epsilon \frac{\lambda_{\star}}{2 h} \delta_{r r}\left|\delta \mathcal{D} u_{r}\right|_{\lambda}^{2}+\frac{h \lambda_{\star}}{2 \epsilon} \delta_{r r}\left|\delta u_{r}\right|_{\Lambda}^{2} \\
& \leq \frac{\epsilon \lambda_{\star} \mathfrak{L}}{2 h} \delta_{r r}\left\|\mathcal{D} u_{r}\right\|^{2}+\frac{h \lambda_{\star} \mathfrak{L}}{2 \epsilon} \delta_{r r}\left\|u_{r}\right\|^{2},
\end{aligned}
$$

joined to 15 . Consequently 17 gets the form

$$
\frac{1}{h^{2}}\left|\mathcal{D} u_{s}\right|^{2}+\frac{1}{h} \delta_{r r}\left\|\mathcal{D} u_{r}\right\|^{2}+\left\|u_{s}\right\|^{2} \leq c+c_{\star} h \delta_{r r}\left\|u_{r}\right\|^{2}
$$

with a new real non-negative constant $c_{\star}$. The required boundedness for the case $c_{\star}>0$ must be then verified using the discrete Gronwall lemma - cf. 33], p. 26, and [45, p. 99. All needed existence and convergence results, analogous to Section II. can be then derived without substantial difficulties.

\section{Quasi-static case}

Many computational tools in fracture mechanics ignore the 1st and 2nd additional terms in (2), which switches to a quasi-static problem. Clearly the 2nd condition (1) is not applicable. Some estimates from Section II. degenerate, e.g. the 1 st inequality 15 to $0=0$. 
Thus that less regular results in comparison with the above discussed dynamic case can be expected and their derivation cannot be repeated following SectionII. easily.

The remedy is to seek for $u \in W^{1,2,2}\left(I, V, V^{\star}\right)$, instead of $u \in W^{1,2,2}(I, V, H)$. As demonstrated by 26 and 49 , some convergence properties must be studied in dual spaces exploiting selected results of [50], instead of estimates like $(19)$. Both propositions P5) and P6) are applicable here. Moreover, the inequality connected with the trace theorem P3) can be used in its more precise form following 51]. However, the convergence of the above introduced 3 types of Rothe sequences (8) and (9) to the solution of (2) (after obvious slight modifications, ignoring the 2 nd condition (1), etc.) is not violated.

\section{A stronger result for dynamic case}

Some experience from the analysis of the quasi-static case can be helpful to upgrade even the results for the dynamic one. One can observe that the weak formulation (7) of (3), crucial for the convergence of Rothe sequences in Section II. can be replaced by its slightly stronger variant

$$
\begin{aligned}
& (v, \rho \ddot{u})+\beta(v, \rho \dot{u}) \\
& +\alpha(\varepsilon(v), C \varepsilon(\dot{u})) \\
& +(\varepsilon(v),(1-\mathfrak{D}) C \varepsilon(u)) \\
& =(v, f)+\langle v, g\rangle_{\Gamma} \quad \text { on } I
\end{aligned}
$$

avoiding all integrals [.,.] in (7). This seems to be supported by the discrete formulation 12 , containing the 2 nd time differences, corresponding to the 2nd time derivatives in 22, naturally. Nevertheless, one cannot guarantee any reasonable convergence of the sequences of 2nd differences to $u \in W^{1,2,2}(I, V, V)$, suffering from the lack of information on $\ddot{u}$ : we have only $u, \dot{u} \in L^{2}(I, V)$ here.

The remedy for such case is to search for $u \in$ $W^{1,2,2,2}\left(I, V, V, V^{*}\right)$, to reach at least $\ddot{u} \in L^{2}\left(I, V^{*}\right)$. One additional type of Rothe sequence is then needed to construct the analogy of the 1st additive term of 10 properly. The verification of its convergence in $V^{*}$, as well as the identification of limits analogous to 19 , brings certain technical difficulties, referring to the properties of spaces of distributions (cf. [50] again), but most results from Section II. remain true. The authors consider to publish all details of (rather extensive) proofs in a separate mathematical paper.

\section{SOFTWARE IMPLEMENTATION}

The computational scheme 11 for the evaluation of $u_{s}^{m}, s \in\{1, \ldots, m\}$, by Section II. and its variants induced by Section III. refers to the numerical analysis of $m$ elliptic problems of infinite dimension. In practical calculations, instead of $v$ in (11) from an infinitedimensional space $V$ we consider a finite number $n$ of test functions $v_{n}$; the approximation $u_{s}^{n m}$ of $u_{s}^{m}$ from (11) with $n$ unknown parameters can be constructed as their linear combinations. Consequently, step-by-step, we choose $v^{n}=\phi_{i}$ where functions $\phi_{i}$ with $i \in\{1, \ldots, n\}$ generate a basis of certain finite-dimensional space $V^{n}$, approximating $V$ (which can be a subspace of $V$ in a special case), and

$$
u_{s}^{n m}=u_{i s}^{n m} \phi_{i}
$$

holds with unknown parameters $u_{i s}^{n m}$, applying the Einstein summation rule with $i \in\{1, \ldots, n\}$.

Let us remark that an concurrent approach can be based on the implementation of adaptive finite element (or similar) discretization on $\Omega$ as the 1st step, switching the original problem of initial and boundary problem for an hyperbolic system of partial differential equations to a large system of nonlinear ordinary differential equations of evolution on $I$, which must be solved in the 2 nd step. Although the methods of analysis of existence and convergence properties and arguments of relevant proofs differ substantially, the resulting fully discretized computational schemes coming from both approaches may coincide.

\section{A. Adaptive discretization}

Typically $\phi_{i}$ are functions with small compact support, applicable in $\Omega$, as well as on $\Theta, \Gamma$ and $\Lambda$, to create a sparse system of linear algebraic equations, and $u_{i s}^{n m}$ by 23 refer to nodal displacement values. The guarantee of solvability of such system, together with the convergence properties for $n \rightarrow \infty$, depend on certain (semi-)regularity of such decomposition due to the XFEM-based (using the so-called eXtended Finite Element Method) adaptive enrichment functions, namely near geometric singularities.

The benefit of XFEM is the increase of precision of numerical approximations of engineering problems with cracks for a wide class of utilizes functions. Another advantage of this method is the almost independence of a mesh on discontinuities. Whereas 52 relies on certain expanding of conventional FEM, special enrichments of approximation functions are suggested by [53. Two basic variants of XFEM can be distinguished: the singularity-based approach and the phantom node one. For their better understanding (23) some special parameters $a_{j s}^{n m}$ and $b_{k l s}^{n m}$, unlike those well-known from the standard FEM techniques, can be separated from $u_{i s}^{n m}$ to enable the presentation of 23 in the form of 54

$$
u_{s}^{n m}=u_{i s}^{n m} \phi_{i}+a_{j s}^{n m} \phi_{j} \mathcal{H}_{j}\left(\mathcal{F}_{j}\right)+b_{k l s}^{n m} \phi_{k} \mathfrak{F}_{l}
$$

now $n$ means the sum of all admissible indices $i, j$ and pairs $(k, l)$ and $i, j, k, l$ undergo the Einstein summation again. Moreover 24 contains the special sign functions $\mathcal{H}_{j}$ with values \pm 1 only, the crack surface functions $\mathcal{F}_{j}$ referring to all parts of $\Lambda$ and some branch functions $\mathfrak{F}_{l}$ extracted from asymptotic fields; for more details of such functions and instructive examples cf. [55], [56] and [57].

The singularity-based approach by $[58]$ pays special attention to the 3rd additive term of $(24)$, whose specification, namely the reasonable form of $\mathfrak{F}_{l}$ from both the point of view of physical justification and that of practical reliable setting of selected material parameters, can be derived the detailed analysis of various modes of stress 
singularities on crack tips following [59]. Unlike this, the phantom node approach by 60 tries to handle cracking using the 2 nd additive term of (24) only, which offers a chance to include the cohesive properties of $\Lambda$ naturally like 61.

However, such notation, induced by 24), compatible with [27] and 13], does not cover all possible algorithms, in particular the original intrinsic method by [62]. Here we shall apply namely the engineering computational approach of 13$]$ to demonstrate the possibility of effective numerical simulations; the discussion of computational techniques for numerical quadrature on relevant adaptive meshes can be found in 54. Much more references to the advances in XFEM strategies in the last decade are contained in 63], 64], [12 and 65]; especially for some inverse problems of identification of material characteristics including sensitivity analysis see 66 and for the potential coupling of XFEM with BEM, i.e. the boundary element method, utilizing some knowledge of (semi-)analytical solutions, cf. 67].

\section{B. Illustrative examples}

The test task is a relatively simple body with an a priori crack of a circular shape. A uniform load was applied to the surface of this a priori crack, and thus the formation of the following cracks emanating from this stress concentrator is assumed using XFEM. The basic calculation system was the commercial software Abaqus 2018, into which a user subroutine in the Fortran 90 language was implemented, realizing the modelling of matrix damage using exponential law, based on the planar element CPE4. It is based on the possibility of user procedures in Abaqus. In principle, it allows you to recalculate the stress distribution in the body or modify the properties of the element, or create a new element. This means that the tested tasks use built-in procedures for solving the finite element method, or modification of the built-in XFEM procedure. At present, there is a need to create a separate code independent of the commercial system.

The following basic input data corresponding to reinforced cement paste: the Young modulus $E=3.2 \mathrm{GPa}$, the Poisson ratio $\mu=0.3$ and the tensile strength 10 $\mathrm{MPa}$ were used for this task. For $3 \mathrm{~mm}$ long metal fibres, the Young modulus $E=190 \mathrm{GPa}$ and the same Poisson ratio $\mu=0.3$ were used.

All figures show some typical distributions of principal stresses in loaded specimens under the plain strain assumption. Their original colours are: red 10-50 MPa, yellow 4-10 MPa, green 2-4 MPa, blue 0.5-2 MPa. Figure 1 demonstrates creation and propagation of a crack using standard (without special modification) XFEM in a cement paste. The stress is initially evenly concentrated on the surface of the hole, due to the different shear modules, the crack spreads logically as presented in right figure. In the following Figure 2, reinforcing metal fibres are introduced into the structure, the first figure shows a situation where there is one fibre in the vicinity of the concentrator, the next figure shows a situation for two fibres.
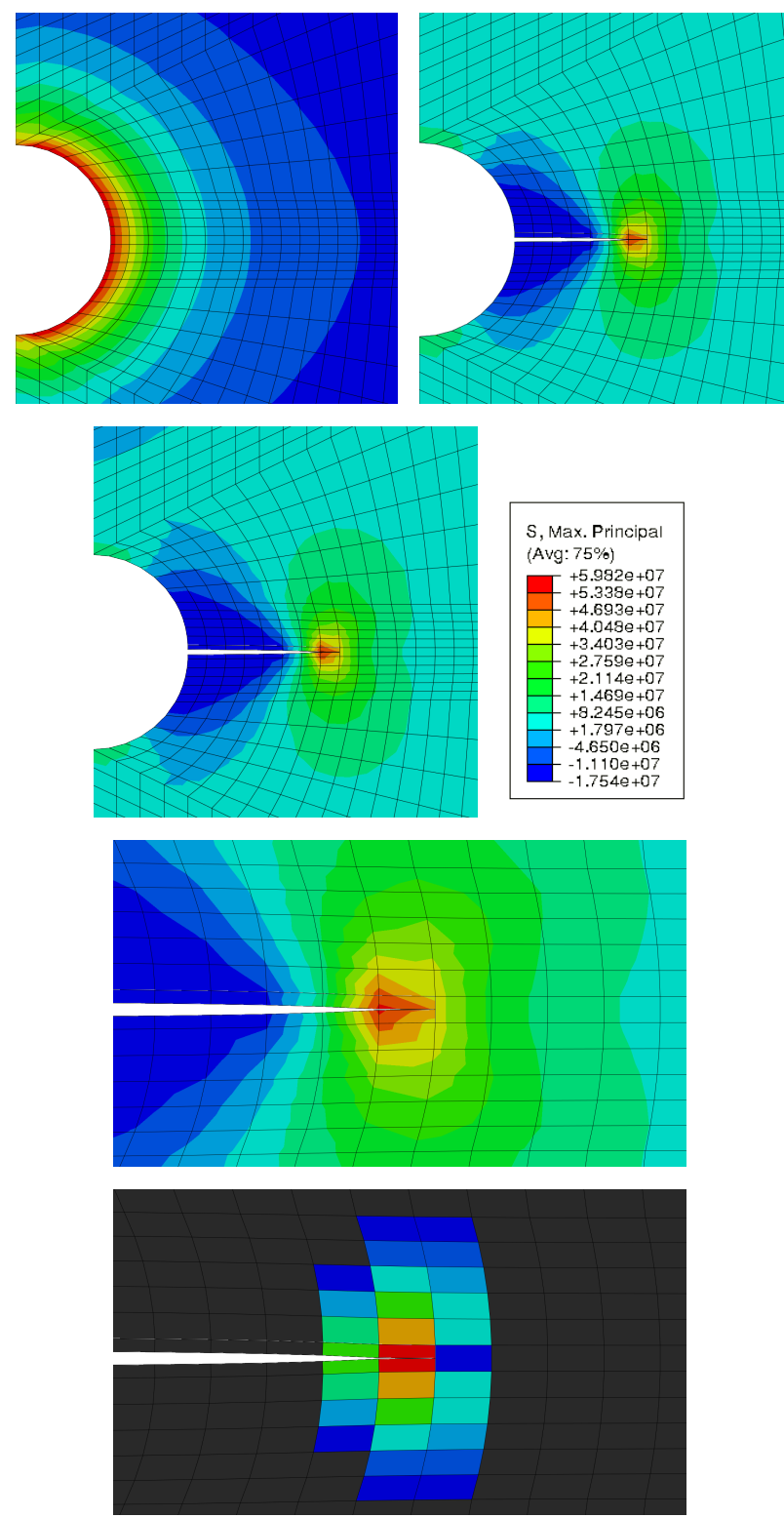

Fig. 5: Nonlocal damage model maximum principal stress with the evaluation of damage factor by [21], applying the exponential formula from [41], close to 2 fibres.

Figure 1 shows the characteristic stress distribution in front of the crack tip, the field is dominant in the direction of tension. The material is isotropic and the highest stress is above the direction of crack propagation; i.e. in fact, delamination occurs in front of the whole crack. The detailed situation is shown in Figure 1 at the bottom. The main stress level for the first two images is selected as 1e7 MPa. The critical area is practically symmetrical on both sides in front of the whole crack. The following Figure 2 shows the effect of fibre shading. Nevertheless, the crack hits the fibre and tries to get around it. Usually the growth of the crack is stopped when the fibre (obstacle) is reached. There is no significant difference for the case of 1 or 2 fibres in the critical area (relation to the unit cell). However, the importance of fibres increases with their concentration. 
The left part of Figure 3 documents how the stress distribution ahead the crack tip is determined when the nonlocal approach is used for computing stress field ahead the crack tip. The right part of Figure 3 attempts to clearly illustrate the algorithm used to calculate the stress concentration in front of the crack tip (an instructive simplified scheme). This picture has a schematic character, as it indicates how the tension is calculated with the help of a non-local approach. In the tested cases, due to the density of the mesh, the stress is calculated only from the following surrounding finite elements. For more accurate analysis, it is necessary to use a finer finite element mesh and calculate the performance for 3-4 layers.

The fundamental question is from what distance from the crack front it is appropriate to calculate the stress distribution in front of the crack front. It should be noted that the results presented in this article are based on experience with the test task. The aim was and is to test the alternatives of individual steps of the algorithm in connection with proper mathematical analysis. In the case of a practical case, we will look for feedback in the physical approach and in general consideration of the size of the elementary cell, which decides on the transfer of the properties of the microstructure to the macrostructure. In general, the size of an elemental cell is determined by about six factors (microcracks, fibers, etc.) that cause the crack to form and grow.

The influence of nonlocal stress calculation is quite clearly observed in the following Figure 4, in the vicinity of the stress concentrator (hole) are two fibres, using the evaluation of damage factor in the exponential form by [21, referring to 41]. If we compare this situation with2, a markedly different character of crack propagation can be observed. The crack is perpendicular to the stress. The effect of fibre may be less than might be expected. Figures 4 and 5 are describing the same situation, but the principle stress and Mises stress are used for comparison and understanding of micromechanisms of fracture.

The decisive factor is the damage factor, which is really dependent on the stress concentration in front of the whole crack. The damage factor is then only a tool for modelling crack propagation. However, the combination with the non-local factor makes it possible to better describe the behaviour of the material, which more clearly characterizes the course of the stress in front of the whole crack represented by the maximum principal stress.

The cleavage fracture is a sequential process involving crack nucleation and propagation. In most materials nucleation occurs at brittle boundary particles (such as inhomogeneities, fibres, etc.) in consequence of stress concentration. Due to microstructural inhomogeneities volume sampling effects play an important role in quantifying the large scatter of fracture data. Reference volume for many theories for brittle fracture initiation are saying that size of this volume and maximum principal stress in this volume are the most important parameters not only for correct material response and numerical modelling using the finite element method.
Finally Figure 6attempts to work with smeared fibres for comparison. The influence of fibres is implemented in the behaviour of the whole structure here, as certain homogenization approach - cf. 9 .
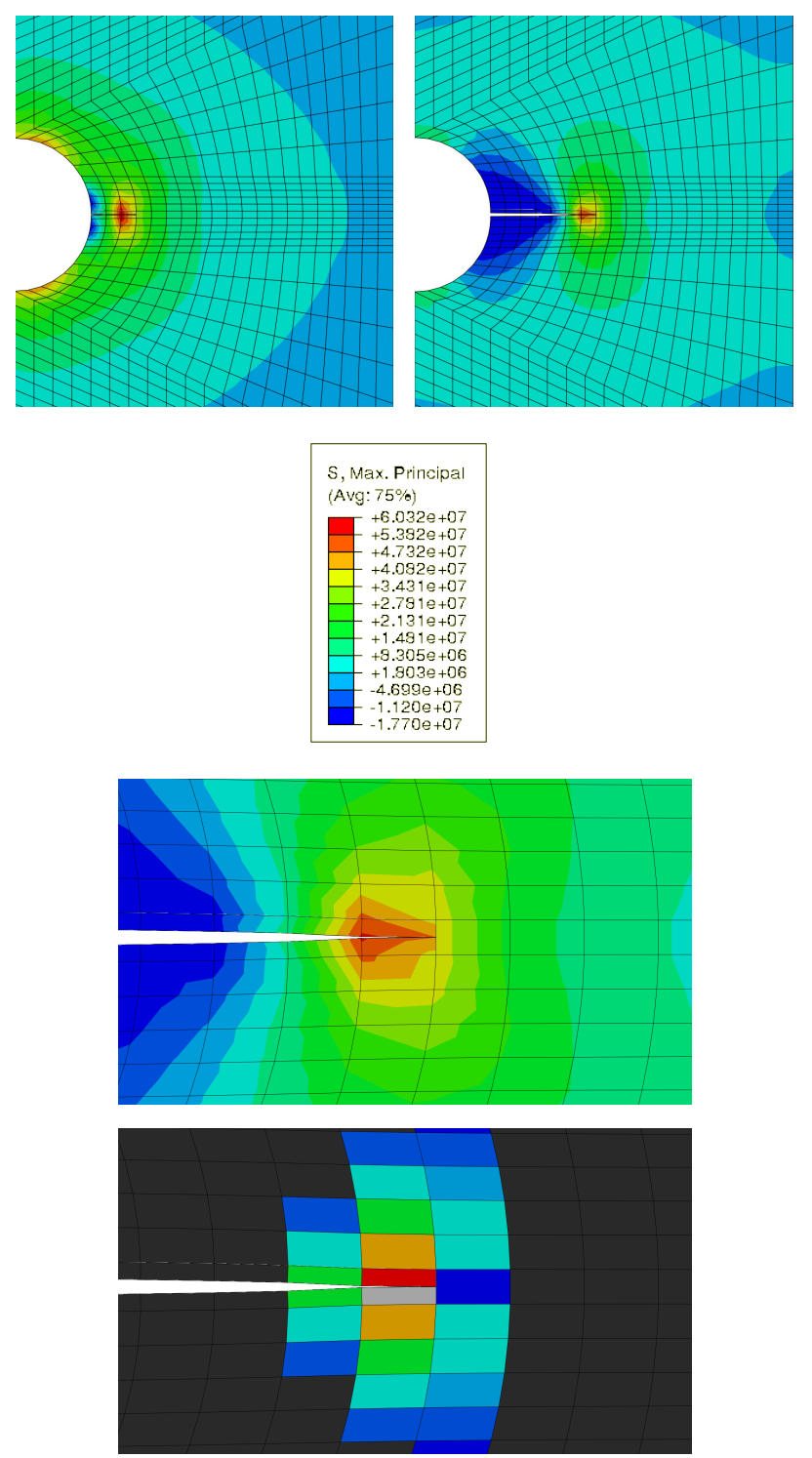

Fig. 6: Computation for a regularized material structure containing smeared fibres.

\section{Conclusion}

Most computational algorithms predicting fracture of brittle and quasi-brittle materials and composites refer to some principles of classical mechanics, but apply ad hoc approaches in their final forms. Queer and mutually incompatible results lead to their seeking for various heuristic macroscopic models with numerous parameters, coming from laboratory experiments, whose reasonable setting needs advanced statistical or soft computing approaches. Unlike them, this paper demonstrates the possibility of well-posed physical and mathematical formulation of a relevant deterministic problem, supported by the constructive design of convergent sequences of approximate solutions, based i) on the method of discretiza- 
tion of time (of Rothe sequences), ii) on the (extended) finite element method, iii) on certain regularization due to the nonlocal strain / stress considerations, following the Eringen model, iv) on the evaluation of cohesive properties of crack interfaces. The related computational approach covers both the micro- and macro-cracking phenomena, applicable to the dynamic case, as well as to the quasi-static one.

Nevertheless, rather strong mathematical and computational simplifications of physical reality are involved here. Some of them have been introduced to avoid readeer-unfriendly technical difficulties. Substantial restrictions occur in still other simplifications, namely in both nonlinear terms (damage factor, cohesive interface): a) the requirement $\varsigma>0$ forbids (even local) complete loss of stiffness due to micro-cracking, whose lifelikeness would be controversial because of the a priori strain-stress linearizations, b) macro-cracks are allowed on a finite number of (potential) cohesive surfaces only, whereas some XFEM algorithms promise to predict them (nearly) everywhere. The reasonable design of $\omega$ for (6), related to a), is also not trivial, as well as that of $\lambda$ for (20), connected with b). Certain inspiration for a proper finite-strain formulation can be found in 68], p. 303, in confrontation with [69] and 70], handling important particular problems.

The above sketched limitations of the presented approach can be interpreted as motivations for future research. Its possible aim of high practical importance can be the development and verification of the computational tool for prediction of brittle and quasi-brittle behaviour of structural components from fibre reinforced composites under mechanical loads, with methodology based on the physical model incorporating such processes as elastic and plastic deformation, crack initiation and propagation in a matrix and debonding or rupture of fibres. As already mentioned, the goal cannot be achieved by writing user procedures in the Abaqus commercial system alone. As the next step, creation of special FEM library inside MATLAB environment is being prepared.

\section{ACKNOWLEDGEMENT}

This work is supported by the project of specific university research at Brno University of Technology (Czech Republic), FAST-S-20-6294.

\section{REFERENCES}

[1] Y. Sumi, Mathematical and Computational Analyses of Cracking Formation. Tokyo: Springer, 2014.

[2] A. Acharya, A possible link between brittle and ductile failure by viewing fracture as a topological defect, Comptes Rendus Mecanique 348 (2020), submitted preprint, pp. 1-12.

[3] J. Vala, Structure identification of metal fibre reinforced cementitious composites, Algoritmy - 20th Conference on Scientific Computing in Podbanské (2016), Proceedings, STU Bratislava, 2016, pp. 244253.
[4] K. Park, G. H. Paulino and J. R. Roesler, Cohesive fracture model for functionally graded fiber reinforced concrete,. Cem. Concr. Res. 40 (2010), 956965.

[5] R. Brighenti and D. Scorza, Numerical modelling of the fracture behaviour of brittle materials reinforced with unidirectional or randomly distributed fibres, Mech. Mater. 52 (2012), 12-27.

[6] A. Cerrone, P. Wawrzynek, A. Nonn, G. H. Paulino and A. Ingraffea, Implementation and verification of the Park-Paulino-Roesler cohesive zone model in 3D, Int. J. Fract. 120 (2014), 26-42.

[7] J. G. Sanjayan, A. Nazari and H. Pouraliakbar, FEA modelling of fracture toughness of steel-reinforced geopolymer composites, Materials and Design 76 (2015), 215-222.

[8] R. Brighenti, A. Carpinteri, A. Spagnoli and D. Scorza, Cracking behaviour of fibre-reinforced cementitious composites: a comparison between a continuous and a discrete computational approach. Eng. Fract. Mech. 103 (2013), 103-114.

[9] M. Jirásek, Damage and smeared crack models, in: Numerical Modeling of Concrete Cracking (G. Hofstetter and G. Meschke, eds.). Udine: Springer: CISM International Centre for Mechanical Sciences 532, 2011, pp. 1-49.

[10] A. Edalat-Behbahani, J.A. O. Barros and A. Ventura-Gouveia, Three-dimensional plasticdamage multidirectional fixed smeared crack approach for modelling concrete structures, Int. J. Solids Struct. 115-116 (2017), 104-125.

[11] M. Moradi, A. R. Begherieh and M. R. Esfahani, Constitutive modeling of steel fiber-reinforced concrete, Int. J. Damage Mech. 29 (2020), 388-412.

[12] X. Li, W. Gao and W. Liu, A mesh objective continuum damage model for quasi-brittle crack modelling and finite element implementation, Int. J. Damage Mech. 28 (2019), pp. 1299-1322.

[13] M. Kaliske, H. Dal, R. Fleischhauer, C. Jenkel and C. Netzker, Characterization of fracture processes by continuum and discrete modelling, Comput. Mech. 50 (2012), pp. 303-320.

[14] X. T. Su, Z.J. Yang and G. H. Liu, Monte Carlo simulation of complex cohesive fracture in random heterogeneous quasi-brittle materials: a 3D study, Int. J. Solids Struct. 47 (2010), pp. 2336-2345.

[15] J. Eliáš, M. Vořechovský, J. Skoček and Z.P. Bažant, Stochastic discrete meso-scale simulations of concrete fracture: comparison to experimental data, Eng. Fract. Mech. 135 (2015), pp. 1-16.

[16] M. Moradi, A. R. Begherieh and M.R. Esfahani, Constitutive modeling of steel fiber-reinforced concrete, Int. J. Damage Mech. 29 (2020), pp. 388-412.

[17] P. Ray, Statistical physics perspective of fracture in brittle and quasi-brittle materials, Phil. Trans. $R$. Soc. A 377 (2019), pp. 20170396/1-13.

[18] R. W. Macek and S. A. Silling, Peridynamics via finite element analysis, Finite Elem. Anal. Des. 43 (2007), pp. 1169-1178. 
[19] E. Emmrich and D. Puhst, Measure-valued and weak solutions to the nonlinear peridynamic model in nonlocal elastodynamics, Nonlinearity 28 (2015), pp. $285 / 1-25$.

[20] A. Javili, R. Mosarata and E. Oterkus, Peridynamics review, Math. Mech. Solids 24 (2019), pp. 37143739 .

[21] C. Giry, F. Dufour and J. Mazars, Stress-based nonlocal damage model, Int. J. Solids and Struct. 48 (2011), pp. 3431-3443.

[22] Y. Z. Povstenko, The nonlocal theory of elasticity and its application to the description of defects in solid bodies, Journal of Mathematical Sciences 97 (1999), pp. 3840-3845.

[23] A. C. Eringen, Theory of Nonlocal Elasticity and Some Applications. Princeton University Press, 1984, technical report 64 .

[24] A. C. Eringen, Nonlocal Continuum Field Theories. New York: Springer, 2002.

[25] M. Jirásek, Nonlocal theories in continuum mechanics, Acta Polytechnica 44/5-6 (2004), pp. 16-34.

[26] J. Vala and V. Kozák, Computational analysis of quasi-brittle fracture in fibre reinforced cementitious composites, Theor. Appl. Fract. Mech. 107 (2020), pp. 102486/1-8.

[27] M. G. Pike and C. Oskay, XFEM modeling of short micro-fiber reinforced composites with cohesive interfaces, Finite Elem. Anal. Des. 106 (2005), pp. 16 31.

[28] V. Kozák and Z. Chlup, Modelling of fibre-matrix interface of brittle matrix long fibre composite by application of cohesive zone method, Key Engineering Materials 465 (2011), pp. 231-234.

[29] L. Bouhala, A. Makradi, S. Belouettar, H. KieferKamal and P. Fréres, Modelling of failure in long fibres reinforced composites by X-FEM and cohesive zone model, Composites Part B 55 (2013), pp. 352361.

[30] V. Kozák, Z. Chlup, P. Padělek and I. Dlouhý, Prediction of the traction separation law of ceramics using iterative finite element modelling, Solid State Phenomena 258 (2017), pp. 186-189.

[31] X. Li and J. Chen, An extensive cohesive damage model for simulation arbitrary damage propagation in engineering materials, Comput. Methods Appl. Mech. Eng. 315 (2017), pp. 744-759.

[32] K. Rektorys, The Method of Discretization in Time and Partial Differential Equations. Dordrecht: D. Reidel, 1982.

[33] T. Roubíček, Nonlinear Partial Differential Equations with Applications. Basel: Birkhäuser, 2013.

[34] A. Bermúdez de Castro, Continuum Thermomechanics. Basel: Birkhäuser, 2005.

[35] N. S. Ottosen and M. Ristinmaa, Thermodynamically based fictitious crack/interface model for general normal and shear loading, Int. J. Solids and Struct. 50 (2013), pp. 3555-3561.

[36] N. Nakamura, Extended Rayleigh damping model, Frontiers if Built Environment 2 (2016), pp. 14/1-
13.

[37] M. Lazar, G. A. Maugin and E. C. Aifantis, On a theory of nonlocal elasticity of bi-Helmholtz type and some applications, Int. J. Solids and Struct. 43 (2006), pp. 1404-1421.

[38] Z. Gao, L. Zhang and W. Yu, A nonlocal continuum damage model for brittle fracture, Eng. Fract. Mech. 189 (2018), pp. 481-500.

[39] L. Dlouhý and S. Pouillon, Application of the design code for steel fibre-reinforced concrete into finite element software, Beton TKS 20/2 (2002), pp. 8-13.

[40] P. Havlásek, P. Grassl and M. Jirásek, Analysis of size effect on strength of quasi-brittle materials using integral-type nonlocal models, Eng. Fract. Mech. 157 (2016), pp. 72-85.

[41] R. H. J. Peerlings, R. de Borst, W, A. M. Brekelmans and M. Geers, Gradient enhanced damage modelling of concrete fracture, Int. J. Numer. Anal. Methods Geomech. 3 (1998), pp. 323-342.

[42] G. Pijaudier-Cabot and J. Mazars, Damage models for concrete, in: Lemaitre Handbook of Materials Behavior Models (J. Lemaitre, ed.), Section 6.13, pp. 500-512. London: Academic Press, 2001.

[43] A. Evgrafov and J. C. Belido, From nonlocal Eringen's model to fractional elasticity, Mathematics and Mechanics of Solids 24 (2019), pp. 1935-1953.

[44] S. Altan, Existence in nonlocal elasticity, Arch. Mech. 41 (1989), pp. 25-36.

[45] P. Drábek and J. Milota, Methods of Nonlinear Analysis, Basel: Birkhäuser, 2013.

[46] O. M. Besov, Embedding of Sobolev Spaces and properties of the domain, Mathematical Notes (Matematicheskie Zametki) 96 (2014), pp. 326-331.

[47] A. Cianchi and V. Mazya, Sobolev inequalities in arbitrary domains, Adv. Math. 293 (2016), pp. 644696.

[48] A. Gogatishvili, J. Neves and B. Opic, Characterization of embeddings of Sobolev-type spaces into generalized Hölder spaces defined by $L^{p}$-modulus of smoothness, Journal of Functional Analysis 276 (2019), pp. 636-657.

[49] J. Vala, Remarks to the computational analysis of semilinear direct and inverse problems of heat transfer, Thermophysics - 24th International Conference in Smolenice (2019), AIP Conference Proceedings 2170, 2019, pp. 020023/1-6.

[50] J. K. Bunkure, Lebegue- Bochner spaces and evolution triples, Int. J. Math. Appl. 7 (2019), pp. 4152.

[51] C. O. Horgan, Eigenvalue estimates and the trace theorem, J. Math. Anal. Appl. 69 (1979), pp. 231-242.

[52] T. Belytchko and T. Black, Elastic crack growth in finite elements with minimal remeshing, Int. J. Numer. Methods Eng. 45 (1999), pp. 601-620.

[53] I. Babuška and J. M. Melenk, The partition of unity method, Int. J. Numer. Methods Eng. 40 (1997), pp. $727-758$.

[54] M. Jedlička, V. Rek and J. Vala, On automation 
of XFEM computations considering general shapes of cracks in terms of the stress analysis of spatial structures, Procedia Structural Integrity 23 (2019), pp. $445-450$.

[55] M. Jirásek, Numerical modeling of strong discontinuities, Revue française de génie civil 6 (2002), pp. 1133-1146.

[56] Z. H. Teng, F. Sun, S. C. Wu, Z. B. Zhang, T. Chen and D. M. Liao, 2018. An adaptively refined XFEM with virtual node polygonal elements for $d y$ namic crack problems, Comput. Mech. 62 (2018), pp. 1087-1106.

[57] H. D. Huynh, M. N. Nguyen, G. Cusatis, S. Tanaka and T. Q. Bui, A polygonal XFEM with new numerical integration for linear elastic fracture mechanics, Eng. Fract. Mech. 213 (2019), pp. 241-263.

[58] G. C. Sih, Crack tip mechanics based on progressive damage of arrow: hierarchy of singularities and multiscale segments, Theor. Appl. Fract. Mech. 51 (2009), pp. 11-32.

[59] F. Zhu, X. Ji, P. He and B. Yheng, On stress singularity at crack tip in elasticity, Result in Physics 13 (2019), pp. 102210/1-7.

[60] J.-H. Song, P. M. A. Areiras and T. Belytchko, A method for dynamic crack and shear band propagation with phantom nodes, Int. J. Numer. Methods Eng. 67 (2006), pp. 868-893.

[61] J. Reiner, M. Veidt, M. Dargush and L. Gross, A progressive analysis of matrix cracking-induced delamination in composite laminates using an advanced phantom node method, J. Compos. Mater. 51 (2016), pp. 2933-2947.

[62] T.-P. Fries and T. Belytschko, The intrinsic XFEM: a method for arbitrary discontinuities without additional unknowns, Int. J. Numer. Methods Eng. 68 (2006), pp. 1358-1385.

[63] A. R. Khoei, Extended Finite Element Method: Theory and Applications. New York: J. Wiley \& Sons, 2015.

[64] M. A. Bennania, A. El Akkadb, A. Elkhalfia, Mixed finite element method for linear elasticity in a cracked domain, WSEAS Transactions on Applied and Theoretical Mechanics 9 (2014), pp. 167-178.

[65] R. F. Swati, L. H. Wen, H. Elahi, A. A. Khan and S. Shad, Extended finite element method (XFEM) analysis of fiber reinforced composites for prediction of micro-crack propagation and delaminations in progressive damage: a review, Microsyst. Technol. 25 (2019), pp. 747-763.

[66] F. Hokeš, M. Hušek, J. Kala and F. Král, Predicting the load-carrying capacity of reinforced concrete structural element, WSEAS Transactions on Applied and Theoretical Mechanics 12 (2017), pp. 1-10.

[67] I. Enescu, Some researches regarding stress intensity factors in crack closure problems, WSEAS Transactions on Applied and Theoretical Mechanics 13 (2018), pp. 187-192.

[68] A. Mielke and T. Roubíček, Rate-Independent Systems. New York: Springer, 2015.
[69] G. Lazzaroni, Quasistatic crack growth in finite elasticity with Lipschitz data, Ann. Mat. Pura Appl. 190 (2011), pp. 165-194.

[70] G. Dal Maso and G. Lazzaroni, Crack growth with non-interpenetration: a simplified proof for the pure Neumann problem, Discrete Contin. Dyn. Syst. 31 (2011), pp. 1219-1231.

\section{CONTRIBUTION OF INDIVIDUAL AUTHORS}

Jiří Vala elaborated the physical and mathematical formulation of the problem including its formal verification. Vladislav Kozák was responsible for all needed computational tools and results and their engineering interpretations.

Petra Jarošová contributed to the design and testing of particular computational algorithms.

\section{Creative Commons Attribution License 4.0 (Attribution 4.0 International, CC BY 4.0)}

This article is published under the terms of the Creative Commons Attribution License 4.0 https://creativecommons.org/licenses/by/4.0/deed.en US 